\title{
Chiral Phase Transition in Linear Sigma Model with Nonextensive Statistical Mechanics
}

\author{
Ke-Ming Shen, Hui Zhang, De-Fu Hou, Ben-Wei Zhang, and En-Ke Wang \\ Key Laboratory of Quark \& Lepton Physics (MOE) and Institute of Particle Physics, Central China Normal University,
} Wuhan 430079, China

Correspondence should be addressed to Ben-Wei Zhang; bwzhang@mail.ccnu.edu.cn

Received 9 September 2017; Accepted 7 November 2017; Published 29 November 2017

Academic Editor: Edward Sarkisyan-Grinbaum

Copyright (C) 2017 Ke-Ming Shen et al. This is an open access article distributed under the Creative Commons Attribution License, which permits unrestricted use, distribution, and reproduction in any medium, provided the original work is properly cited. The publication of this article was funded by SCOAP $^{3}$.

From the nonextensive statistical mechanics, we investigate the chiral phase transition at finite temperature $T$ and baryon chemical potential $\mu_{B}$ in the framework of the linear sigma model. The corresponding nonextensive distribution, based on Tsallis' statistics, is characterized by a dimensionless nonextensive parameter, $q$, and the results in the usual Boltzmann-Gibbs case are recovered when $q \rightarrow 1$. The thermodynamics of the linear sigma model and its corresponding phase diagram are analysed. At high temperature region, the critical temperature $T_{c}$ is shown to decrease with increasing $q$ from the phase diagram in the $(T, \mu)$ plane. However, larger values of $q$ cause the rise of $T_{c}$ at low temperature but high chemical potential. Moreover, it is found that $\mu$ different from zero corresponds to a first-order phase transition while $\mu=0$ to a crossover one. The critical endpoint (CEP) carries higher chemical potential but lower temperature with $q$ increasing due to the nonextensive effects.

\section{Introduction}

Quantum Chromodynamics (QCD) is a basic theory of describing the strong interactions among quarks and gluons, the fundamental constituents of matter. More and more attentions have already been attracted to the QCD phase transition both theoretically and experimentally $[1-8]$. Though experimental studies and lattice Monte-Carlo simulations have made it possible to research on the phase diagram quantitatively, there still remains uncertainty at high baryon density region [9]. Consequently, the phase transition is also a vital topic in high-energy physics, where the thermal vacuum created by heavy-ion collisions differs from the one at zero temperature and chemical potential [7]. In order to study certain essential features of it, the linear sigma model has been proposed to illuminate the restoration of chiral symmetry and its spontaneous breaking [8].

Near the phase transition boundary, one should be cautious when using the Boltzmann-Gibbs (BG) statistics for the appearance of critical fluctuations due to a large correlation length [10]. It is of interest to investigate the phase transition in the formalism beyond conventional BG statistical mechanics. Recently nonextensive statistics firstly proposed in [11] has attracted a lot of attention and discussions [12]. In this formalism, instead of the exponential function, a generalized $q$-exponential function is defined as $[11,12]$

$$
\exp _{q}(x):=[1+(1-q) x]^{1 /(1-q)},
$$

where the parameter $q$ is called the nonextensive parameter, which accounts for all possible factors violating assumptions of the usual BG case. Its inverse function is also listed [11, 12]

$$
\ln _{q}(x):=\frac{x^{1-q}-1}{1-q}
$$

Both of them return to the usual exponential and logarithm function with $q \rightarrow 1$.

The purpose of this paper is to clarify the nonextensive effects on physical quantities of the chiral phase transition in the generalized linear sigma model. We focus on the situations where both of temperature and chemical potential 
are not vanished, which then indicates the influence of the Tsallis distribution on the whole phase diagram in the $(T, \mu)$ plane. Whereas the nonextensive parameter $q$ is still a phenomenological parameter [12], not only the case of $q>1$ but also $q<1$, in this work, is computed. For comparisons, we have presented discussions on this issue for finite temperature but vanishing chemical potential $[13,14]$. Given the consistency of nonextensive generalizations with the initial BG approaches, we also list the results of $q=1$ which were investigated [15]. We close our researches with the comparisons to the nonextensive Nambu Jona-Lasinio model ( $q$-NJL model) $[16,17]$ of the critical endpoint (CEP), whose location is still the hot topic for experiments as well as its theoretical researches $[3,5]$.

This paper is organized as follows. In Section 2 we introduce the theoretical framework, where the nonextensive $q$-linear sigma model is stated. Their consequences for various thermodynamic quantities with different nonextensive parameters, q, are explored in Section 3; more detailed discussions on the results are also contained. Section 4 is our brief summary and outlook.

\section{Theoretical Framework}

Within the linear sigma model, the chiral effective Lagrangian with quark degrees of freedom reads $[15,18,19]$

$$
\begin{aligned}
\mathscr{L}= & \bar{\psi}\left[i \gamma^{\mu} \partial_{\mu}-g\left(\sigma+i \gamma_{5} \vec{\tau} \cdot \vec{\pi}\right)\right] \psi \\
& +\frac{1}{2}\left(\partial_{\mu} \sigma \partial^{\mu} \sigma+\partial_{\mu} \vec{\pi} \cdot \partial^{\mu} \vec{\pi}\right)-U(\sigma, \vec{\pi}),
\end{aligned}
$$

where $\psi=(u, d)$ stands for the spin-1/2 two flavors' light quark fields and the scalar field $\sigma$ and the pion field $\vec{\pi}=$ $\left(\pi_{1}, \pi_{2}, \pi_{3}\right)$ together form a chiral field $\Phi=(\sigma, \vec{\pi})$, with its potential

$$
U(\sigma, \vec{\pi})=\frac{\lambda^{2}}{4}\left(\sigma^{2}+\vec{\pi}^{2}-v^{2}\right)^{2}-H \sigma
$$

Considering the obvious symmetry breaking term $H \sigma=0$, $\mathscr{L}$ is invariant under chiral $\mathrm{SU}(2)_{L} \times \mathrm{SU}(2)_{R}$ transformations. The chiral symmetry is spontaneously broken in the vacuum with the expectation values: $\langle\sigma\rangle=f_{\pi}$ and $\langle\vec{\pi}\rangle=0$, where $f_{\pi}=93 \mathrm{MeV}$ is the pion decay constant. By the partially conserved axial vector current (PCAC) relation [15], the quantity $v^{2}=f_{\pi}^{2}-m_{\pi}^{2} / \lambda^{2}$ with the constant $H=f_{\pi} m_{\pi}^{2}$, where $m_{\pi}=138 \mathrm{MeV}$ is the pion mass. The coupling constant $\lambda^{2}$ is fixed as 20 by $m_{\sigma}^{2}=2 \lambda^{2} f_{\pi}^{2}+m_{\pi}^{2}$, where $m_{\sigma}=600 \mathrm{MeV}$ is the sigma mass. Another coupling constant $g$ is usually determined by the requirement of the constituent quark mass in vacuum, $M_{\mathrm{vac}}=g f_{\pi}$, which is about $1 / 3$ of the nucleon mass, leading to $g \approx 3.3$. [15]

In order to investigate the temperature $T$ and the chemical potential $\mu$ dependence in this model, let us consider a system of both quarks and antiquarks in the thermodynamical equilibrium. Here quark chemical potential $\mu \equiv \mu_{B} / 3$. And the grand partition function goes like

$$
\begin{aligned}
\mathscr{Z} & =\operatorname{Tr} \exp \left[-\frac{\mathscr{H}-\mu \mathcal{N}}{\mathrm{T}}\right] \\
& =\int \mathscr{D} \bar{\psi} \mathscr{D} \psi \mathscr{D} \sigma \mathscr{D} \vec{\pi} \exp \left[\int_{x}\left(\mathscr{L}+\mu \bar{\psi} \gamma^{0} \psi\right)\right],
\end{aligned}
$$

where $\int_{x} \equiv i \int_{0}^{1 / T} d t \int_{V} d^{3} \vec{x}$ with $V$ being the volume of the system.

Thus the grand canonical potential can be obtained

$$
\Omega(T, \mu)=-\frac{T}{V} \ln \mathscr{Z}=U(\sigma, \vec{\pi})+\Omega_{\bar{\psi} \psi}
$$

with the (anti)quark contribution being

$$
\begin{aligned}
& \Omega_{\bar{\psi} \psi}(T, \mu)=-v \int \frac{d^{3} \vec{p}}{(2 \pi)^{3}}\left\{E+T \ln \left[1+e^{-(-\mu+E) / T}\right]\right. \\
& \left.+T \ln \left[1+e^{-(\mu+E) / T}\right]\right\}
\end{aligned}
$$

where $v=12$ is the internal degrees of freedom of quarks and $E=\sqrt{p^{2}+M^{2}}$ is the valence (anti)quark energy, with the mass of constituent (anti)quark defined as

$$
M^{2}=g^{2}\left(\sigma^{2}+\vec{\pi}^{2}\right) .
$$

Here the first divergent term of $E$ is absorbed in the coupling constant in the results which comes from the negative energy states of the Dirac sea.

It is inadequate to apply naively the BG statistics in such a system; critical fluctuations of energy and particle numbers will appear as well as a large correlation. In order to investigate the phase transition of systems departing from the classical thermal equilibrium, the nonextensive statistics [11] are introduced. The so-called Tsallis entropy and density matrix are given, respectively, as $S_{q}=k_{B} \operatorname{Tr}\left(\rho-\rho^{q}\right) /(q-$ 1) and $\rho=\exp _{q}(-E / T) / Z_{q}$, where $k_{B}$ is the Boltzmann constant (set to 1 for simplicity next), $q$ describes the degree of nonextensivity, and $Z_{q}$ is the corresponding generalized partition function.

Recently these generalized statistics have been of great interest theoretically [20-22] and widely applied in many fields [23-26]. In the following, we investigate the linear sigma model within the nonextensive statistics. Firstly we rewrite the partition function of (5) as

$$
\begin{aligned}
\mathscr{Z}_{q} & =\operatorname{Tr} \exp _{q}\left[-\frac{\mathscr{H}-\mu \mathcal{N}}{T}\right] \\
& =\int \mathscr{D} \bar{\psi} \mathscr{D} \psi \mathscr{D} \sigma \mathscr{D} \vec{\pi} \exp _{q}\left[\int_{x}\left(\mathscr{L}+\mu \bar{\psi} \gamma^{0} \psi\right)\right],
\end{aligned}
$$


where the $q$-exponential is seen in (1). Considering the $q$ thermodynamics [27], we have

$$
\begin{gathered}
\Omega_{\bar{\psi} \psi}(T, \mu, q)=-\frac{T}{V} \ln _{q} \mathscr{E}_{q}-U(\sigma, \vec{\pi}) \\
=\sum_{n} \sum_{p}\left\{\ln _{q}\left[\beta^{2}\left(E_{n}^{2}+(E-\mu)^{2}\right)\right]\right. \\
\left.+\ln _{q}\left[\beta^{2}\left(E_{n}^{2}+(E+\mu)^{2}\right)\right]\right\} .
\end{gathered}
$$

Before carrying it out, we give out the generalized identities with respect to $q$-sums and integrals

$$
\begin{aligned}
\ln _{q}[ & \left.\beta^{2}\left(E_{n}^{2}+(E \pm \mu)^{2}\right)\right] \\
= & \int_{1}^{\beta^{2}(E \pm \mu)^{2}} \frac{d \theta^{2}}{\left(\theta^{2}+(2 n+1)^{2} \pi^{2}\right)^{q}} \\
& +\ln _{q}\left[1+(2 n+1)^{2} \pi^{2}\right]
\end{aligned}
$$

and the generalized sum over $n$, in our assumptions,

$$
\begin{aligned}
& \sum_{n} \frac{1}{\left(\theta^{2}+(2 n+1)^{2} \pi^{2}\right)^{q}} \\
& \quad \approx \frac{1}{\theta}\left(\frac{1}{2}-\frac{1}{\left(\exp _{2-q}(\theta)+1\right)^{q}}\right),
\end{aligned}
$$

where $E_{n}=(2 n+1) \pi T$ is used and the index $2-q$ appears because of the duality

$$
\begin{aligned}
& \exp _{q}(-x) \cdot \exp _{2-q}(x) \\
& \quad=[1-(1-q) x]^{1 /(1-q)} \cdot[1+(q-1) x]^{1 /(q-1)}=1 .
\end{aligned}
$$

Integrating over $\theta$ and dropping terms that are independent of $\beta$ and $\mu$, we finally obtain

$$
\begin{aligned}
& \Omega_{\bar{\psi} \psi}(T, \mu, q)=-v \int \frac{d^{3} \vec{p}}{(2 \pi)^{3}}\{E \\
& +T \ln _{q}\left[1+\exp _{q}\left(-\frac{E-\mu}{T}\right)\right] \\
& \left.+T \ln _{q}\left[1+\exp _{q}\left(-\frac{E+\mu}{T}\right)\right]\right\} .
\end{aligned}
$$

In our calculations within the mean-field approximation, we follow $[15,28]$ where the expectation value of the pion field is set to zero; $\vec{\pi}=0$. By solving the gap equation

$$
\left.\frac{\partial}{\partial \sigma} \Omega_{\bar{\psi} \psi}(T, \mu, q)\right|_{\sigma=\sigma_{V}}=0
$$

the value for constituent (anti)quark mass $M=g \sigma_{V}$ can be determined, which will be also affected by different nonextensive parameters, $q$. Here we have replaced $\sigma$ and $\vec{\pi}$ in the exponent by their expectation values in the mean-field approximation.

With such a $q$-thermal effective potential, we then explore the nonextensive effects on the physical quantities, as well as the phase transition, in the linear sigma model. The numerical results will be shown in the next section.

\section{Results and Discussion}

In virtue of the fact that there still exist fierce controversies over the possible interpretations of the nonextensive parameter $q$, we shall discuss the nonextensive effects in the $q$-linear sigma model for both the $q>1$ and $q<1$ case. Meanwhile, we give out the result of $q=1$ as the baseline for better understanding.

It is worthy to note that the value of nonextensive parameter $q$ cannot be much smaller than 1 since, in the expression of (14), the corresponding generalized exponential

$$
\exp _{q}\left(-\frac{E+\mu}{T}\right):=\left[1-(1-q) \frac{(E+\mu)}{T}\right]^{1 /(1-q)}
$$

where the part of the base should be larger than $0: 1-(1-$ $q)((E+\mu) / T)>0$; namely,

$$
q>1-\frac{T}{(E+\mu)} .
$$

Thus some upper limitation of energy of the integral in (7) should be given in case of divergence when $q<1$. On the other hand, too much smaller values of $q$ are not necessary to be computed physically during our investigation on the nonextensive effects on the phase transition. Therefore, here we just list the results of $q=1.1,1.05,0.95$ and $q=1$ as baseline.

We start our discussions with presenting in Figure 1 the resulting thermodynamical potential $\Omega$ as a function of $M$, the constituent (anti)quark mass. Different $q$ evidently results in a large change of the thermodynamical potential which shows that the effects caused by nonextensivity are quite strong whether the quark chemical potential vanishes or not.

In Figure 1(a), the potentials with different $q$ are shown for $T=148 \mathrm{MeV}$ and $\mu=0$. Locations of its minimum become smaller when $q$ gets larger. This means that, in the case of high temperature and low density, correlations with the nonextensive $q$-version shift the chiral condensation toward smaller $M$. On the other hand (in Figure 1(b)), at low temperature but high density $(T=40 \mathrm{MeV}$ and $\mu=$ $286.8 \mathrm{MeV}$ ), the gap of potential between local (near $M=0$ ) and global (far from $M=0$ ) vacuum also increases as $q$ increases. It is worthy to note that, as seen in Figure 1(b), different $q$ nearly does not affect the position of global vacuum which should have nothing to do with the model itself.

It is instructive to plot the $q$-effects on the constituent (anti)quark mass $M$ under the temperature dependencies as well as the chemical potential dependencies, which are clearly shown in Figure 2. For the $T$-dependence $(\mu=0)$, the values of $M$ change continuously with the temperature $T$, which describes a typical crossover transition, while, for the $\mu$-dependence (where we set $T=40 \mathrm{MeV}$ ), it shows a jump over the values of $M$, demonstrating a first-order phase transition.

Figure 2(a), at low density, indicates that the temperature dependence of $M$ for $q \neq 1$ is quite similar to the case of $q=1$, the usual BG situation. Both the minimum and maximum 


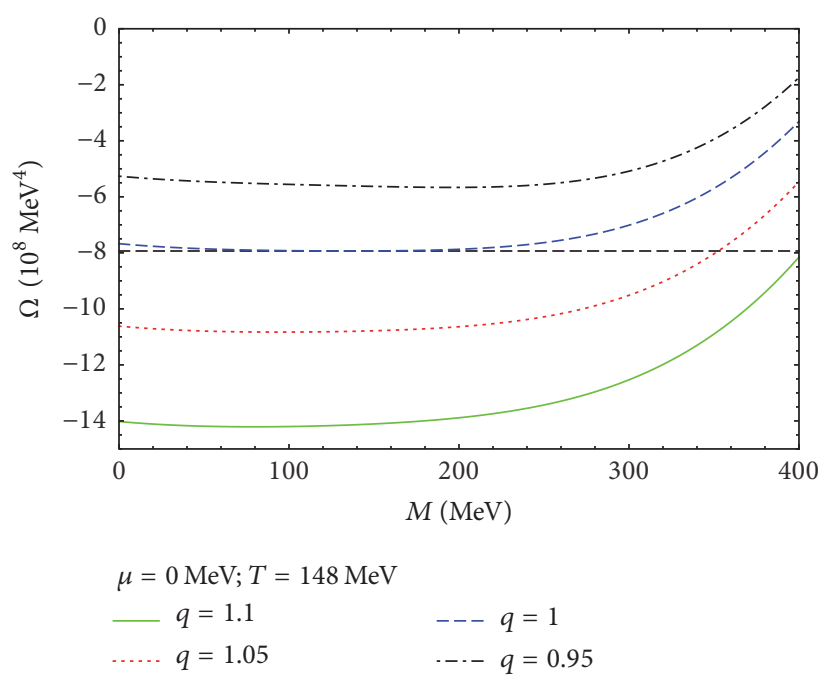

(a)

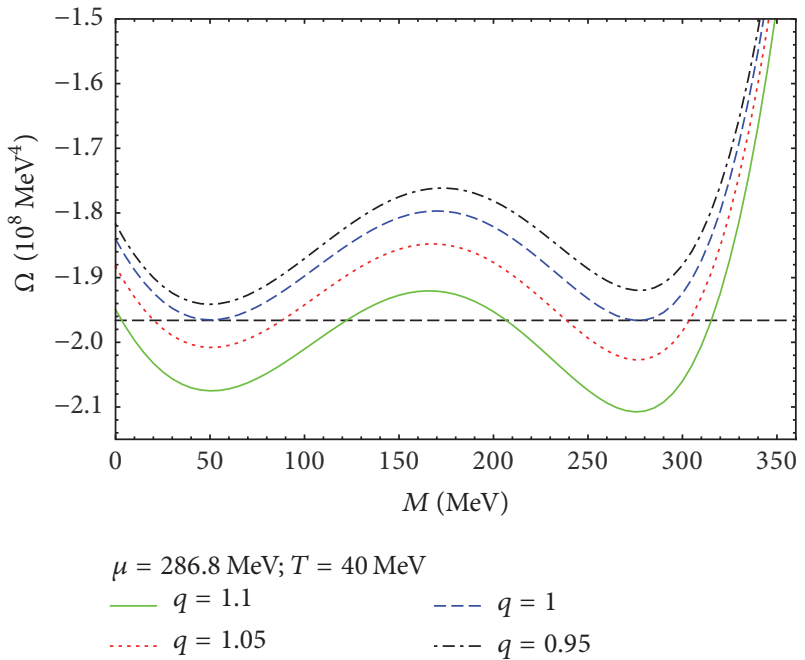

(b)

Figure 1: The thermodynamical potentials $\Omega$ (cf. (14) and (7)), with respect to the constituent (anti)quark mass $M$. (a) $T=148 \mathrm{MeV}$ and $\mu=0$. (b) $T=40 \mathrm{MeV}$ and $\mu=286.8 \mathrm{MeV}$. We compare our results using the parameters near the phase boundaries. Both of them are analysed for $q=1.1, q=1.05$, and $q=0.95$ with the case of $q=1$ as comparisons.

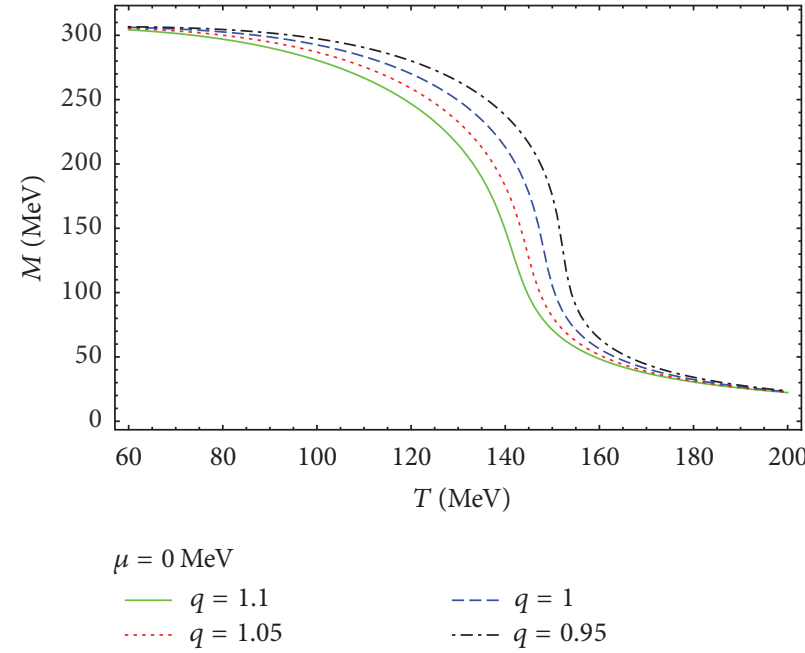

(a)

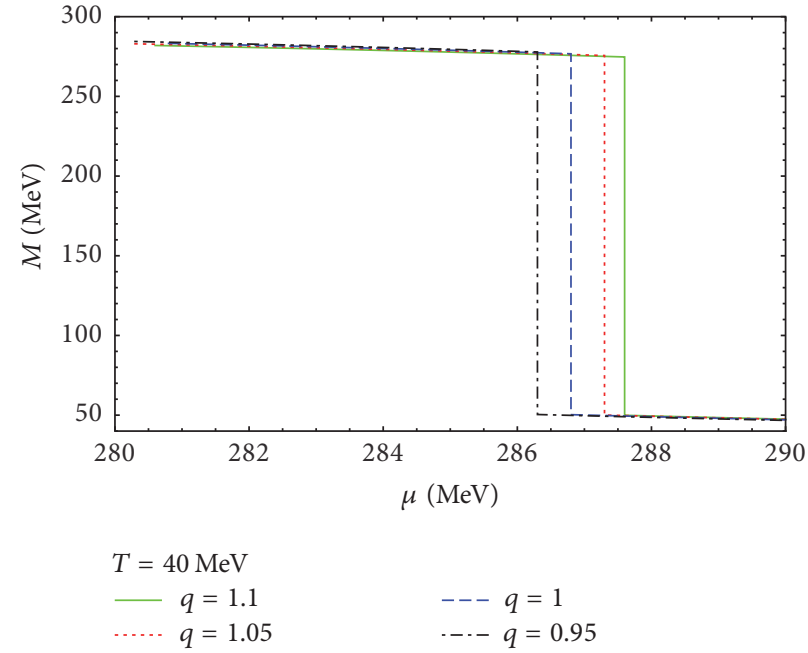

(b)

Figure 2: The constituent (anti)quark mass $M$ as functions of the temperature $T$ at $\mu=0$ (a) and the chemical potential $\mu$ at $T=40 \mathrm{MeV}$ (b) for different $q$ as follows: $q=1.1, q=1.05$, and $q=0.95$ as well as $q=1$.

of $M$ keep the same values for different $q$. Nevertheless, the behaviour of all curves tells us that high temperature is required to restore the chiral symmetry for small $q$, which agrees with the results of $[13,14]$.

At the same time, for the low temperature case, Figure 2(b) illustrates the $\mu$-dependence of the constituent (anti)quark mass in the nonextensive linear sigma model for different nonextensive parameter $q$, which is not done in [13, 14]. One easily observes an analogous pattern characteristic to the above, while, for the $\mu$-dependence, increasing $q$ will also increase the value of phase transition chemical potential when $T=40 \mathrm{MeV}$ is fixed. Moreover, for both of the cases, it is deserved to be mentioned that only the system near the chiral phase transition is well affected by nonextensive statistics.

In statistical physics, the critical properties of a thermodynamic system can be explored by studying the fluctuations of various observables. Particularly, the fluctuations of the order parameter probe the order of the phase transition and the position of a possible critical end point.

Then the negative partial derivative of $M$ with respect to temperature $T$ holding chemical potential $\mu$ constant, the susceptibility $\chi$, is also investigated in this nonextensive linear sigma model, which describes the fluctuation of constituent (anti)quark mass. From the results seen in Figure 3, one can expect that, at the low density $(\mu=0)$, the location of peak 


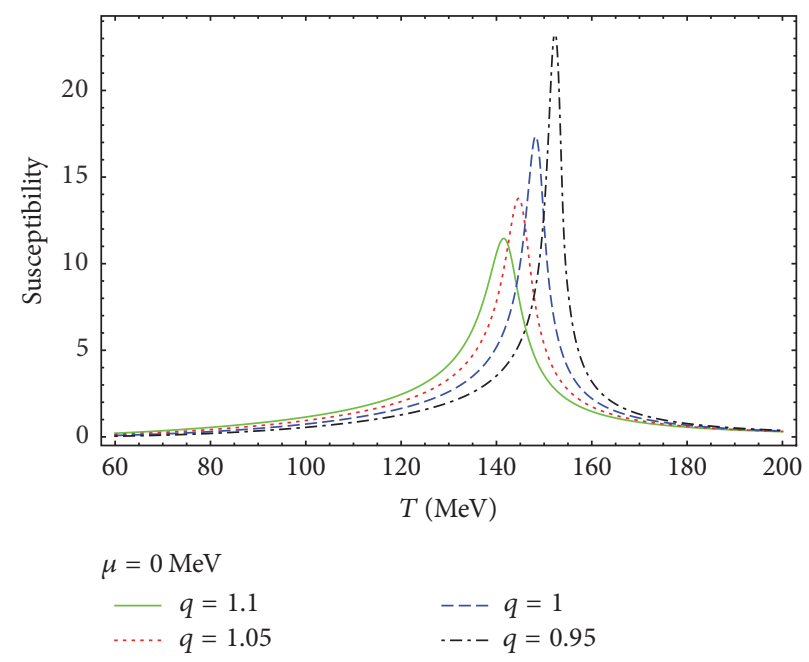

FIGURE 3: The nonextensive effects on susceptibility $\chi=-\left.(\partial M / \partial T)\right|_{\mu}$ with temperature $T$ at $\mu=0$ are shown for various $q$ values as shown in previous figures $(q=1.1, q=1.05$, and $q=0.95$ as well as $q=1$ for baseline).

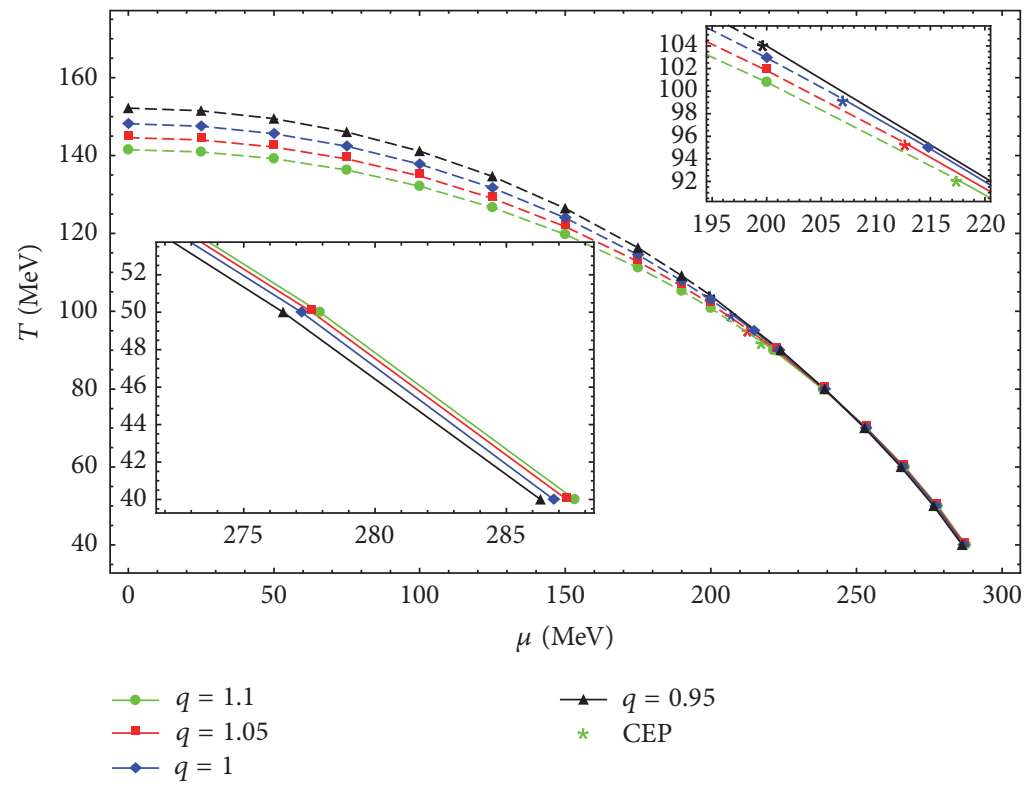

Figure 4: Phase diagram of the $q$-linear sigma model in the $(T, \mu)$ plane for various $q$ values. The results are plotted for four different values of $q$ with the vicinity of the $q$-dependent CEP and the low temperature part of the curves enlarged in the inset. For more details, the dashed line stands for crossover transition and the solid one the first-order transition. CEPs are shown as star points.

of susceptibility $\chi$, as well as its own value, moves to the lower values of temperature $T$ for larger $q$. This indicates that, with larger $q$, the critical temperature $T_{c}$ gets smaller, which supports the fact that the nonextensive parameter $q$ describes the departure of system from the conditions of BG situation.

Here we add a few remarks to better understand the results. Nonextensive dynamics develop the linear sigma model through the (anti)quark number distribution functions. These functions are connected with the thermal potential $\Omega_{\bar{\psi} \psi}(T, \mu, q)$ of (14), which modifies the fluctuations of fermions. The $q$-dependent chiral condensation can be obtained after solving out the gap equation in (15). From Figure 2(a), we can see that its shape with respect to $T$ is strongly affected by the nonextensive parameter $q$. More specifically, $q$ introduces differences of system itself from the usual BG one which decrease the values of critical temperature $T_{c}$, seen in Figure 3.

In order to explore the chiral phase transition in the $q$ linear sigma model more specifically, we also present the phase diagrams (seen in Figure 4) based upon the analysis above. It is easily seen that, indeed, at high temperature and low density region, it exhibits a crossover transition in the $(T-\mu)$ plane for different nonextensive parameters of $q$, with smaller nonextensive parameter $q$ expanding the relative values of critical temperature and chemical potential. Meanwhile, a first-order phase transition is shown at low 
temperature but high density region. And all the critical lines correspondingly develop differently, where larger $q$ increases the position of $T_{c}$ at the same $\mu_{c}$.

As for the critical endpoint (CEP), which is located between the two kinds of phase transition, larger $q$ occurs at higher chemical potential but lower temperature, which is also seen in the results of $q$-NJL model $[16,17]$. This is because systems from fewer particles will encounter a larger value of $q$, whose phase transition takes place with higher number density in turn.

\section{Summary and Outlook}

To summarize, we have calculated the nonextensive thermodynamics of the chiral phase transition in the linear sigma model, to account for the sensitivity of the meanfield theory of the linear sigma model to the departure from the usual BG statistics. By the $q$-version, we have obtained generalized relations of the grand canonical potential $\Omega$, the chiral condensation $M$, and susceptibility $\chi$. Before that, we also analysed the values of nonextensive parameter $q$ and reasonably considered the cases of $q=1.1,1.05,0.95$ as well as $q=1$.

Furthermore, we have investigated two scenarios, $\mu \neq 0$ and $\mu=0$, respectively, which, as mentioned, correspond to different physical situations: a first-order and a crossover transition. For the studies of $\mu=0$, it is found to be in agreement with the results obtained in $[13,14]$. Besides, we discover that different values of $q$ only influence the quantities near the phase boundary. This also proves that it is valuable and desirable to discuss the nonextensive effects on the chiral phase transition.

As expected, the observed nonextensive effects of both the potential $\Omega$ and the mass $M$ lead to the fact that higher values of $q$ shift all to an earlier state with other parameters fixed. In other words, the internal divergence from the classical thermal equilibrium really impacts the chiral phase transition. This is more illuminated in the phase diagrams of $(T, \mu)$ plane correspondingly. The CEP (see Figure 4) reveals a clear variation with different nonextensive parameters of $q$, namely, holding higher chemical potential but lower temperature with $q$ increasing, which agrees with $[16,17]$. As for the critical line in the diagram, as shown in Figure 4, q-effects derive different trends of it on the first-order and crossover phase transitions, whose physical mechanism needs from us more attention and investigations next.

Finally, it is worthy to mention that since CEP is still indistinct experimentally, our work may provide a possible intensive study of locating the CEP in high-energy physics [6]. Meanwhile, by comparing the results with experimental data, our researches could be of help to deeply understand the physical explanation of the Tsallis nonextensive parameter $q$, which is also what we will pay attention to in the future.

\section{Conflicts of Interest}

The authors declare that they have no conflicts of interest.

\section{Acknowledgments}

This research is partly supported by the Ministry of Science and Technology of China (MSTC) under the "973" Project nos. 2014CB845404 and 2015CB856904(4) and by NSFC under Grant nos. 11435004, 11322546, 11375070, and 11521064.

\section{References}

[1] A. Bazavov, H.-T. Ding, P. Hegde et al., "QCD Equation of State to $\partial\left(\mu_{B}^{6}\right)$ from Lattice QCD," Physical Review D, vol. 95, article 054504, 2017.

[2] H. Reinhardt and P. Vastag, "Chiral and deconfinement phase transition in the Hamiltonian approach to QCD in Coulomb gauge," Physical Review D: Particles, Fields, Gravitation and Cosmology, vol. 94, no. 10, 2016.

[3] R. A. Lacey, "Indications for a critical end point in the phase diagram for hot and dense nuclear matter," Physical Review Letters, vol. 114, article 142301, 2015.

[4] P. Braun-Munzinger and J. Wambach, "Colloquium: Phase diagram of strongly interacting matter," Reviews of Modern Physics, vol. 81, no. 3, pp. 1031-1050, 2009.

[5] C. S. Fischer, J. Luecker, and C. A. Welzbacher, "Locating the critical end point of QCD," Nuclear Physics A, vol. 931, pp. 774779, 2014.

[6] J. Randrup, "Phase transition dynamics for baryon-dense matter," Physical Review C: Nuclear Physics, vol. 79, no. 5, 2009.

[7] M. Gyulassy and L. McLerran, "New forms of QCD matter discovered at RHIC," Nuclear Physics A, vol. 750, no. 1, pp. 3063, 2005.

[8] M. Abu-Shady and H. M. Mansour, " Quantized linear ," Physical Review C: Nuclear Physics, vol. 85, no. 5, 2012.

[9] P. de Forcrand, "Simulating QCD at finite density," PoS LAT, vol. 010, 2009.

[10] M. Stephanov, K. Rajagopal, and E. Shuryak, "Signatures of the tricritical point in QCD," Physical Review Letters, vol. 81, no. 22, pp. 4816-4819, 1998.

[11] C. Tsallis, "Possible generalization of Boltzmann-Gibbs statistics," Journal of Statistical Physics, vol. 52, no. 1-2, pp. 479-487, 1988.

[12] Lists of many applications of non-extensive statistics are available at http://tsallis.cat.cbpf.br/biblio.htm.

[13] M. Ishihara, "Effects of the Tsallis distribution in the linear sigma model," International Journal of Modern Physics E, vol. 24, article 1550085, 2015.

[14] M. Ishihara, "Chiral phase transitions in the linear sigma model in the Tsallis nonextensive statistics," International Journal of Modern Physics E, vol. 25, article 1650066, 2016.

[15] O. Scavenius, Á. Mócsy, I. N. Mishustin, and D. H. Rischke, "Chiral phase transition within effective models with constituent quarks," Physical Review C, vol. 64, article 045202, 2001.

[16] J. Rozynek and G. Wilk, "Nonextensive effects in the Nambu-Jona-Lasinio model of QCD," Journal of Physics G: Nuclear and Particle Physics, vol. 36, article 125108, 2009.

[17] J. Rozynek and G. Wilk, "Nonextensive Nambu-Jona-Lasinio Model of QCD matter," The European Physical Journal A, vol. 52, no. 13, 2016.

[18] M. C. Birse and M. K. Banerjee, "A chiral soliton model of nucleon and delta," Physics Letters B, vol. 136, no. 4, pp. 284288, 1984. 
[19] M. C. Birse and M. K. Banerjee, "Chiral model for nucleon and delta," Physical Review D: Particles, Fields, Gravitation and Cosmology, vol. 31, no. 1, pp. 118-127, 1985.

[20] E. K. Lenzi, L. C. Malacarne, and R. S. Mendes, "Perturbation and variational methods in nonextensive tsallis statistics," Physical Review Letters, vol. 80, no. 2, pp. 218-221, 1998.

[21] C. Beck, "Dynamical foundations of nonextensive statistical mechanics," Physical Review Letters, vol. 87, no. 18, article 180601, 2001.

[22] F. D. Nobre, M. A. Rego-Monteiro, and C. Tsallis, "Nonlinear relativistic and quantum equations with a common type of solution," Physical Review Letters, vol. 106, no. 14, article 140601, 2011.

[23] T. S. Biro and G. Purcsel, "Non-extensive Boltzmann equation and hadronization," Physical Review Letters, vol. 95, article 162302, 2005.

[24] F. I. Pereira, R. Silva, and J. S. Alcaniz, "Nonextensive effects on the relativistic nuclear equation of state," Physical Review C: Nuclear Physics, vol. 76, no. 1, 2007.

[25] B. Liu and J. Goree, "Superdiffusion and Non-Gaussian Statistics in a Driven-Dissipative 2D Dusty Plasma," Physical Review Letters, vol. 100, no. 5, 2008.

[26] T. S. Biró and E. Molnár, "Fluid dynamical equations and transport coefficients of relativistic gases with non-extensive statistics," Physical Review C: Nuclear Physics, vol. 85, no. 2, 2012.

[27] C. Tsallis, Introduction to Nonextensive Statistical Mechanics: Approaching a Complex World, Springer, New York, NY, USA, 2009.

[28] L. P. Csernai, A. Mocsy, and I. N. Mishustin, "Microscopic model for rapid hadronization of supercooled Quark-Gluon Plasma," Heavy Ion Physics, vol. 3, no. 151, 1996. 

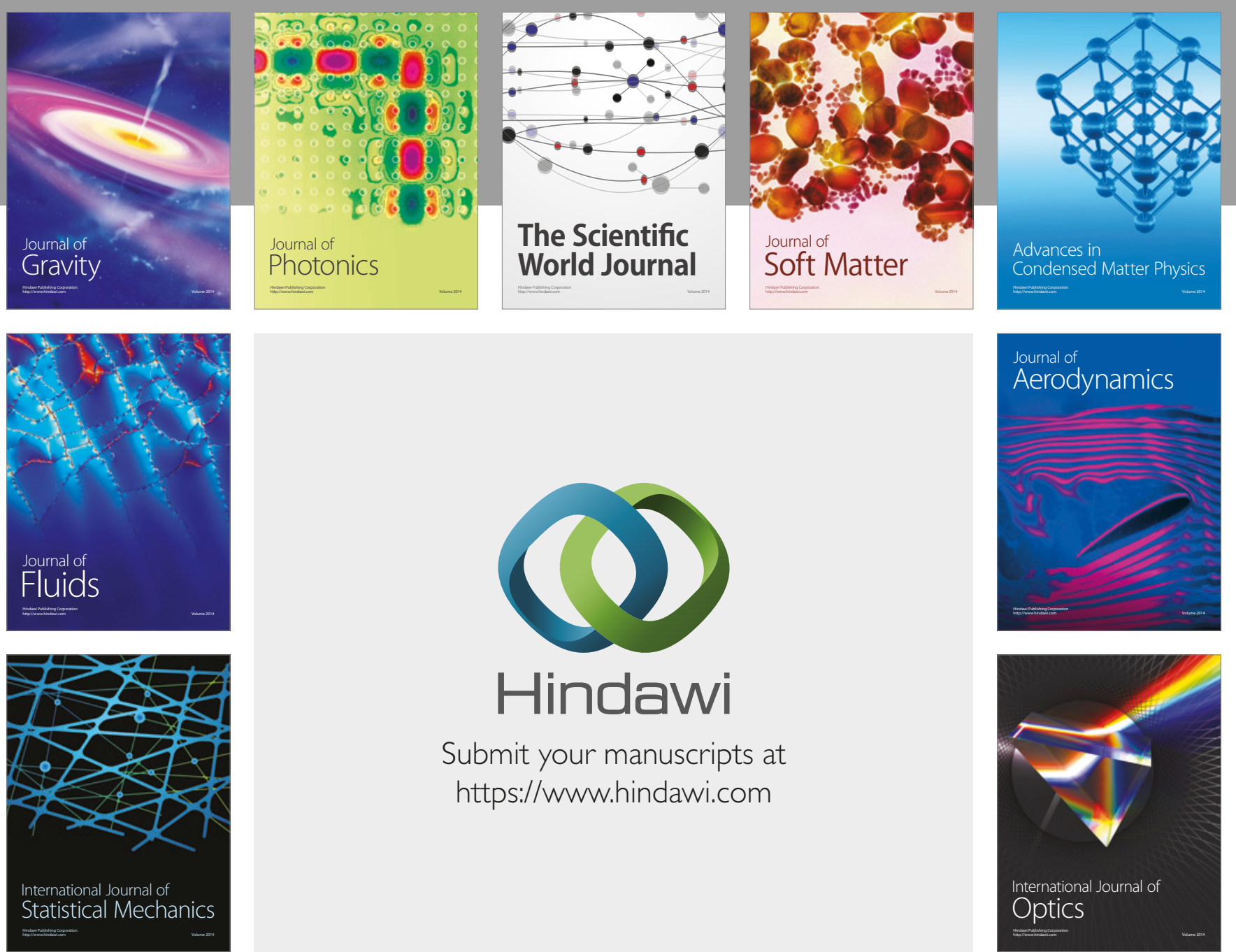

Submit your manuscripts at

https://www.hindawi.com
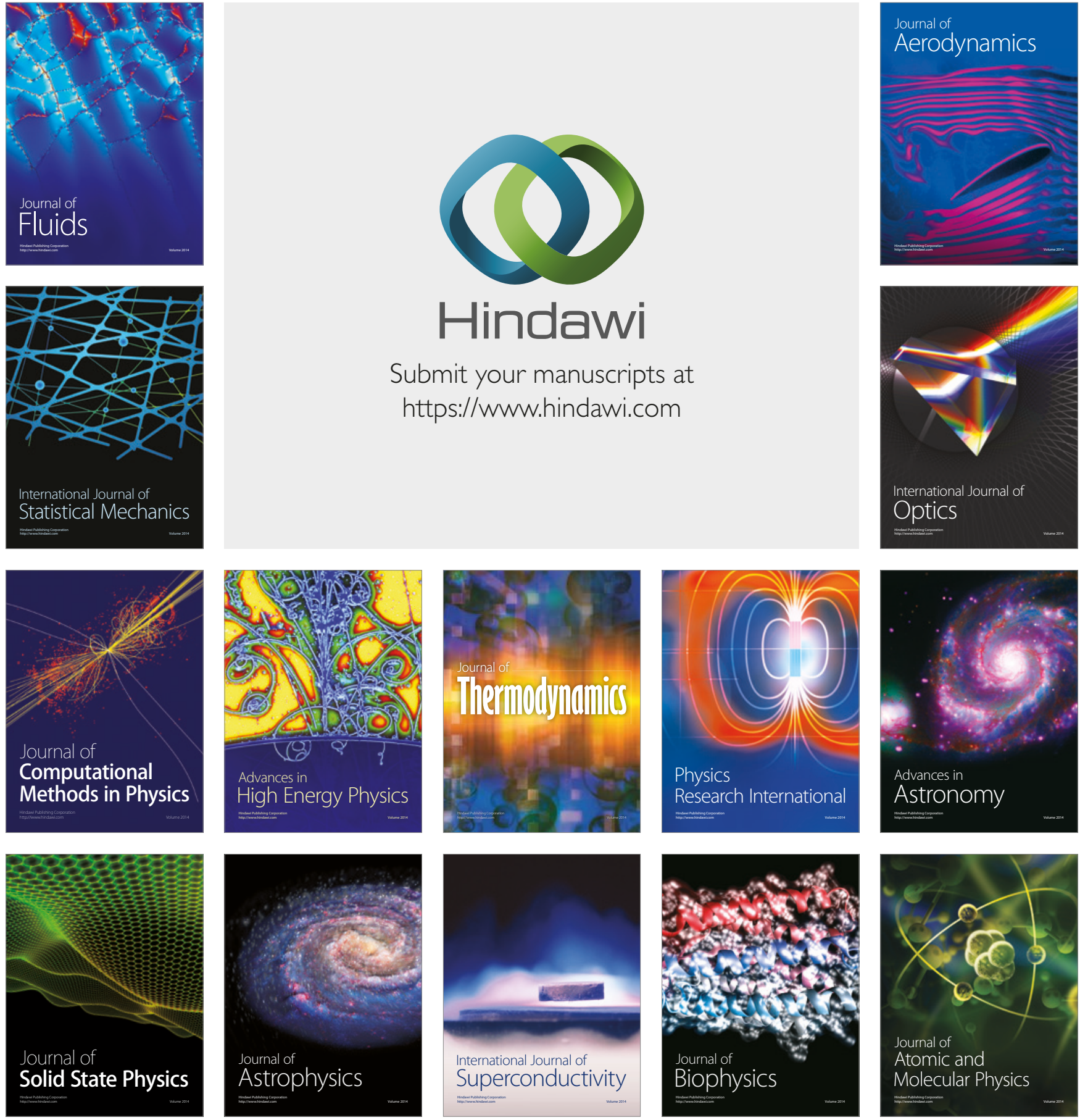